\title{
Supra-regional services for the deaf
}

\section{Epidemiological aspects of prelingual deafness and psychiatry}

Definitions

Prelingual deafness (PLD) has onset before the acquisition and use of spoken language. It may be congenital or early acquired, and encompass profound to partial hearing loss. 'Social deafness' includes people unable to take part in a brisk conversation (Basilier, 1972).

\section{Demography}

Incidence and prevalence are $0.1 \% ; 50,000$ PLD people in England and Wales; only $10 \%$ have one or both deaf parents; $90 \%$ of the children of PLD parents are hearing; or married PLD people, 95\% have a deaf spouse (DHSS, 1988).

Deafness is associated with other disabilities; $28 \%$ of aged between 16-59 who are registered as disabled and live in private housing have a hearing loss, whereas it is $53 \%$ for the disabled of the same age in communal establishments (Martin et al, 1988). It is estimated that $30 \%$ of PLD have other disabilities.

\section{Epidemiology}

Between 1929 and 1966, studies have shown a three to ten-fold over-representation of deaf people in psychiatric hospitals in New York State, England, Germany and Denmark (Remvig, 1969). Average length of stay was vastly greater than for hearing patients (Remvig, 1969; Stein et al, 1975). As recently as 1985 , these findings were replicated in the Netherlands (Timmermans, 1989). Of in-patients, diagnoses were similar in proportion for deaf and hearing (Stein et al, 1975; Rainer et al, 1963), although there is a suggestion that major affective disorders are under-represented. The lifetime risk of schizophrenia in deaf people has been calculated at $2.5 \%$ (v. $1 \%$ in hearing). However, diagnosis can be difficult and analysis of 250 patients seen by a specialist deaf unit has shown (Denmark, 1975): (approximately) 20\% schizophrenia; 20\% other mental illness; $20 \%$ developmental disorders of communication; $20 \%$ behaviour disorder (related to deafness); $20 \%$ other (including organic, diagnosis unclear).

The quality of these statistics is open to question because no operationalised diagnostic criteria were used. Currently, however, there is ongoing work using the Clinical Interview Schedule and a translation of the GHQ-30 to estimate the prevalence of mental disorder in a small sample of the general deaf adult population (Checinski, 1990); initial results indicate a level of disorder somewhat higher than in comparable studies in the hearing.

\section{Psychiatric services for deaf children and adolescents}

Profound prelingual deafness affects one in a thousand children in the United Kingdom. Even with modern hearing aids many of these children will have little useful hearing and will experience great difficulty in acquiring spoken or written English. Nearly $30 \%$ have an additional handicap and $10 \%$ have evidence of brain damage. Only the $10 \%$ of children born to deaf parents (deaf people who use British Sign Language) will be exposed to sign language from birth. Many deaf children of hearing parents will first encounter sign language when they start school, some considerably later. The deaf children who acquire sign language also acquire a new cultural identity, they become deaf.

The prevalence of psychiatric disorder in deaf children has been estimated as being one and a half to five times that in hearing children. In a recent study of deaf adolescents, the rate of psychiatric disorder was $30 \%$. Many deaf children with psychiatric disorders do not receive treatment. Their disorders go unrecognised, often being attributed to their deafness. When deaf children are referred to local child psychiatry services, they are often seen as being untreatable because of difficulties with language and communication.

Young children with delayed speech and functional hearing loss can benefit from the use of signs, either Makaton or 'Baby Talk BSL', in the period before a definitive diagnosis can be made. Signing can be continued as appropriate.

As a consequence of the factors outlined above, the assessment, diagnosis and treatment of psychiatric disorders in deaf children is fraught with difficulty. Deafness is relatively uncommon. A local child psychiatrist will have little opportunity to develop the necessary expertise in sign language and knowledge of deaf culture to assess and treat deaf children. Limited specialist services exist; the two existing Supra-Regional Deaf Units see deaf children but neither consultant psychiatrist is a child psychiatrist. The Nuffield Centre provides a weekly out-patient service but the consultant child psychiatrist does not have specific training in deafness. 
The Springfield Deaf Unit is in the process of establishing a service for deaf children and adolescents. This will initially provide an assessment and consultation service, with limited therapeutic facilities. There is little awareness of the psychiatric problems of deaf children and limited knowledge. The child psychiatry service will also provide teaching and research opportunities to address these problems.

\section{Present services needs}

The previous College position statement on Supra Regional Services for the Deaf was forwarded to the Department of Health in 1986. Although the two original units at Whittingham and Springfield can act as Supra-Regional Centres with provision of clinical services to the North and South-East, there remain areas where deaf patients with psychiatric disorder are isolated from suitable services, such regions whose problems have been identified as West Wales and East Anglia. A third specialist unit is planned in Birmingham as part of the Birmingham and West Midlands Postgraduate Psychiatric Teaching Programme and clinical services. This unit will serve the needs of people at the centre of England. Since it is known that deaf people with mental disorder are likely to need services for a considerable period of time, it is essential that units not only develop links within the locality for long-term care, but also peripatetic support to those referred from regions without a specialised service.

Co-operation in clinical work and senior registrar teaching will also be required in those areas with specialist units for the deaf, with other specialties within psychiatry in order to provide some service for the whole age group.

There still remain a large number of people in the community or now being rehabilitated from longterm residential care whose deafness is not fully recognised and are unable to function to their full potential as a result. In the provision of care plans for people with mental disorders, handicaps and social needs, the group with such unrecognised hearing loss will require specialised services.

\section{Teaching and research}

The units have an essential part in teaching the medical profession from medical students to consultant colleagues about the emotional difficulties experienced by people with hearing loss who seek medical advice. They would also contribute to more specialised teaching on the psychiatric sequelae of deafness.

Further epidemiological research is indicated, but clinical experience has shown a large number of unmet needs in this population.

\section{Special needs staff}

Disorders of communication of all sorts are closely correlated with psychiatric disorders. The care of deaf people with mental health needs demands highly trained personnel with specific communication skills as well as a broad knowledge of psychiatry with particular reference to deaf people and the management of behavioural disorders. The staff ratio will need to be in excess of that required for services for those with complex handicap.

To meet the psychiatric needs of deaf adults and children, there are now three highly motivated senior registrars near the end of their training. This training is highly individual and specialised and should not be allowed to waste.

\section{Membership of the Working Party}

Dr A. Gath (Chairman); Dr N. Kitson; Dr B. Monteiro; Dr P. Hindley; Dr K. Checkinski.

\section{References}

BASILIER, T. (1972) Scandinavian Audiology, 1.2, 55-68.

CHECINSKI, K. (in progress) An Estimate of the Point Prevalence of Psychiatric Disorder in PLD Adults in the General Population.

Denmark, J. C. (1985) A study of 250 patients referred to a department of psychiatry for the deaf. British Journal of Psychiatry, 146, 282-286.

DHSS SOCIAL SERVICES INSPECTORATE (1988) Say It Again. London: HMSO.

MARTIN, J. et al (1988) The Prevalence of Disability among Adults. London: HMSO. Pp. 32, 36.

RAINER, J. D. et al (1963) Family and Mental Health Problems in a Deaf Population. Ch 14, K. Z. Altshuler and J. D. Rainer, pp. 200, 202.

REMvig, J. (1969) Acta Psychiatrica Scandinavica Supplement, 210, pp. 12, 17-18.

StEIN, L. K. et al (1975) Deafness and Mental Health. Ch. 8, K. Altshuler \& S. Abdullah, pp. 101-103.

Timmermans, L. (1989) Proceedings of the 1st European Congress on Mental Health and Deafness. p. 91.

Approved by the Executive and Finance Committee September 1990 